\title{
Location Awareness and Positioning System Based on RFID
}

\author{
Yihang Li, Zhenji Cao, Yuan He \\ Beijing University of Posts and Telecommunications, Beijing102209 \\ huner2011@foxmail.com
}

Keywords: RFID, Location Awareness, Indoor Positioning, Tracking

\begin{abstract}
Location awareness refers to the services related to the location which are provided by the users. And its application can't work without the users' location information. Because the traditional positioning technology can't meet the requirements of location environment and accuracy indoors, the advantages of RFID sensor technology, such as non-contact, non-line of sight and so on make it become the first choice for the indoor positioning technology. This paper emphatically has studied the data processing from the two perspectives of object localization and target tracking, and has taken the positioning algorithm as a key question to carry on a deep discussion.
\end{abstract}

\section{Introduction}

In the information society, the improvement of the efficiency and accuracy of data acquisition is the common focus of each industry. And the RFID wireless identification is undoubtedly a big step in this aspect. Real-time location system is a kind of system which uses electronic device at any time to locate a person or thing. Real-time location system uses wireless remote sensing technology to provide wireless service for the users. It is mainly applied to the assets in the region of network which has already have wireless connectivity for real-time location tracking. The market of RTLS real-time locating systems is still relatively small. So far approximately 1000 systems like these have been implemented around the world. But RTLS real-time location systems will be implemented in the future. The RTLS real-time location and tracking system mainly makes use of unique identification characteristics which the labels act on the objects. It depends on the signal strength of radio frequency communication between the reader and the labels installed on the objects to measure the spatial location of objects. It is mainly used in indoor positioning of the GPS system with the difficulty of application.

\section{The Application of RFID System in the Indoor Environment}

The application of RFID system in the indoor environment can be divided into three aspects:

The labels of supply chain located on the interior object. An important role of RFID label is to replace the bar code technology to apply in each link of the supply chain. And any item in the world will have its unique electronic product code (EPC, Electronic Product Code).

The reference labels located in the indoor environment. This kind of labels are mainly considered as symbol of the location. It provides the reference for the tracking of moving targets. At the same time, it also provides the necessary information of the indoor environment. This kind of labels are usually fixed in the indoor buildings, such as walls. They are widely distributed in an indoor environment. And they provide the location of the signal strength information. And they effectively regulate the effect of dynamic factors in indoor environments for mobile target tracking. Therefore, when we install such kind of labels, we should design the position of the fixed label according to the need of indoor location environment to make them be used to build the wireless signal map of Interior layout.

The active labels located on the moving target. The purpose of using the active labels is to be able to position the moving targets better in the indoor environment. It can be seen that the application of RFID system in indoor environment is very flexible. The RFID label not only acts as the positioning tool in the indoor environment but also provides the corresponding environment or 
the items information. It is not difficult to imagine that afterwards the RFID label will be attached to all the items at home to help the system more intuitive to respond to the needs of users. Of course, all these are done in a reasonable way with the respect the privacy.

\section{k- nearest neighboring location algorithm}

When we use FRID technology to make indoor location, the positioning system needs to offer the information of signal strength which the label travels to the reader. The thought of this paper mainly comes from the LANDMARC system which uses active RFID label to make indoor location.

Suppose that there are $\mathrm{n}$ readers, m reference labels, u mobile labels which are to be tracked in the positioning area. When the reader works in continuous mode, it tests once every 30 seconds with the test scope of 1 8 grade. The signal strength vector of mobile and to-be-tracked label(in the following it is called to-be-tracked label for short) is defined as $S=\left(\mathrm{S}_{1}, \mathrm{~S}_{2}, \cdots, \mathrm{S}_{\mathrm{n}}\right) . S_{i}$ is signal strength of to-be-tracked label, which is detected by the reader-writer i. $i \in(1, n)$. For the reference label, we define the corresponding signal strength vector as $\theta=\left(\theta_{1}, \theta_{2}, \ldots, \theta_{n}\right)$, in which $\theta_{i}$ is signal strength. We use Euclidean geometry method to detect the distance of signal strength. For any tobe-tracked label, we define that:

$$
E_{j}=\sqrt{\sum_{i=1}^{n}\left(\theta_{i}-S_{i}\right)^{2}}, j \in(1, m)
$$

$E_{j}$ is Euclidean distance of signal strength between to-be-tracked label and reference label $r_{j}$. E indicates the position relationship between reference label and to-be-tracked label. For example, if the distance between reference label and to-be-tracked label is closer, the value of $\mathrm{E}$ is smaller. If there are $\mathrm{m}$ reference labels, the to-be-tracked label will have vector value of E. $E=\left(E_{1}, E_{2}, \cdots, E_{m}\right)$.

$$
(x, y)=\sum_{i=1}^{k} W_{i}\left(x_{i}, y_{i}\right)
$$

This algorithm should find the closest neighbor of unknown to-be-tracked label by comparing different $\mathrm{E}$ values. Because these values of $\mathrm{E}$ can only reflect the relationship of labels, we use energy grade value of report to replace signal strength value in equations. When we use k closest coordinates of reference label to locate a unknown label, we call it k- nearest neighbor algorithm. This thought originally exists when there are several neighbors which are closest to the targets, we will track in signal space. Considering the measured signal strength in a certain point may change, so there is no reason to only choose one closest neighbor and give no consideration to other neighboring points. According to the things above, we can get the coordinate of unknown label:

$$
(x, y)=\sum_{i=1}^{k} W_{i}\left(x_{i}, y_{i}\right)
$$

$W_{i}$ is the $\mathrm{i}^{\text {th }}$ weight of neighboring reference label. Weight factor selection is another designing parameter. Suppose $k$ nearest labels have the same weight(for example $W_{i}=1 / k$ ). That will generate certain error. Therefore, the decision of different nearest label weight is also a important problem. Generally speaking, the value of $W_{i}$ depends on $E$ value of every reference label in space. For example, $W_{i}$ is the function of $\mathrm{k}$ nearest $E$ values. We have tried different methods to decide the weight. Here we give the weight equation from experience:

$$
W_{j}=\frac{1 / E_{i}^{2}}{\sum_{i=1}^{k} 1 / E_{i}^{2}}
$$


This method generates the smallest error in most of experiments. In other words, reference label with the smallest $\mathrm{E}$ value has the biggest weight. That explains that signal strength is inversely proportional to the square of the distance.

In order to test the implementation efficiency of system, we use distance error to measure precision of the system. We define position estimation error e, which is the linear distance between actual coordinate $\left(x_{0}, y_{0}\right)$ of to-be-tracked label and calculating coordinate $(x, y)$. The equation is in the following,

$$
e=\sqrt{\left(x-x_{0}\right)^{2}+\left(y-y_{0}\right)^{2}}
$$

After we have the positioning algorithm, one of the following key problems is to find optimal $\mathrm{k}$ value in the algorithm. We choose different $k$ values. When $k$ equals $1,2,3,4,5$, we calculate coordinate of to-be-tracked label respectively. By repeating experiments we find that positioning precision does not increase with the increasing $\mathrm{k}$ value all the time. Therefore, By repeating the experimental demonstration with LANDMARC method we thinks that when k equals 4, we can get a relative higher positioning precision.

\section{The tracking of mobile label}

In this part, we will analyze the problem of tracking mobile label and not analyze problem of positioning fixed label like the way before. Before making indoor tracking, we need to receive RSS wireless map of signal strength, namely, a database that stores signal strength of different indoor positions. During the tracking phase, the system receives signal strength of unknown points, matches with RSS wireless map, and estimates the position by using tracking algorithm.

Data collection of RSS wireless map can be completed in the off-line phase. Database includes 3 basic sheets: RSS basic sheet which stores original signal strength data has collected from different positions; RSS main value sheet which stores the mean value of signal strength; RSS history sheet which stores the history data of signal strength. We can build RSS wireless map by using these 3 sheets.

When we build the map, we can use indoor layout to reduce logic error effectively. If the users move between different floors or walk from one side of wall to the other side, the signal strength should be the same. Indoor layout uses connected graph to represent. And the two vertexes of direct connection indicate the neighboring location. In the connected graph, the shortest route between any 2 points can be obtained with Dijkstra shortest route algorithm.

Processing flow of tracking algorithm is shown in the figure:

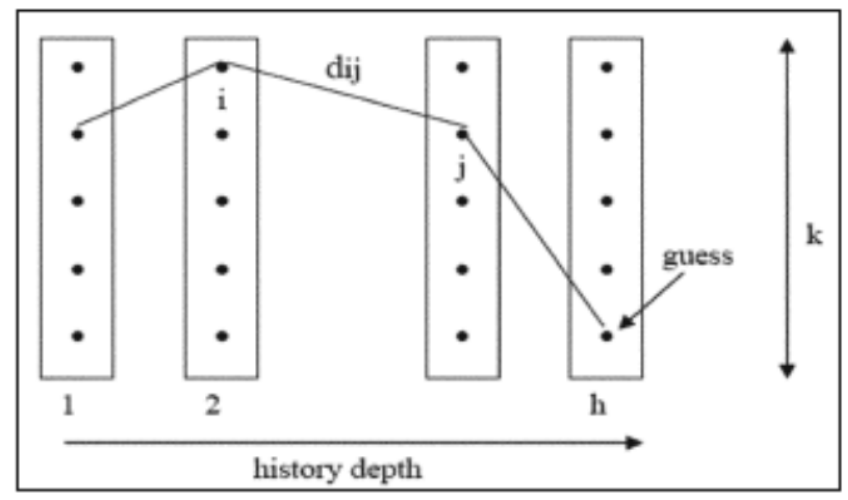

Fig. 1 schematic diagram of tracking algorithm

As is shown in figure 1, processing flow of algorithm is in the following steps:

(1)When active RFID label moves, current signal strength sample is collected periodically.

(2)In RSS wireless map, look for k neighboring points whose Euclidean distance with current signal strength sample is the smallest.

(3)In k nearest history vector, delete the oldest neighboring point and add the newest neighboring point to maintain the length of history vector to be $\mathrm{k}$ all the time. 
(4)Calculate the shortest route between the oldest neighboring point and the newest one. The distance of 2 neighboring points is the physical distance of site layout.

(5)The end point of the shortest route is the current position of active RFID label.

(6)Repeat above steps until the client stops tracking

In the experiment, we find that if the moving speed of users is slower, the tracking effect will be better. In ideal environment, if moving user stays on one unknown point for several seconds, the average positioning error is about 1 meter. If moving user moves slowly without pausing, the average error is about 1.4 meter.

\section{Conclusion}

This paper uses a method which is based on receiving signal strength to make positioning estimation. When we calculate the user's position, we have used Euclidean distance between user's actual position and estimation position as error distance. When we get the positioning process of indoor reference label, we have introduced simple signal propagation model and modified the model by introducing wall attenuation factor WAF; And we have made parameter estimation using linear regression analysis; we have solved the surface equations and calculated to get label position by analyzing Fang algorithm or Chan algorithm. The advantage of this method is that we can obtain accurate position with complex calculation. And we can configure according to the actual need for the reference label indoors. Based on indoor reference label, we have got the indoor mobile label positioning. The advantage of doing in that way is that we can solve the influence of dynamic environment factors on label signal detection. The nearest neighboring algorithm dynamically corrects the corresponding relationship between distance and signal strength. At the same time, simple calculation is greatly helpful to obtain real-time information too.

\section{References}

[1] Garmin Corporation. About GPS. Website, 2001. www.garmin.com/aboutGPS.

[2] Roy Want, Andy Hopper, Veronica Falco et al. The Active Badge Location System. ACM Transactions on Information System.1992.10(1):91-102.

[3] Paramvir Bahl and Venkata N.Padmanabhan. RADAR: An in-building RF-based user location and tracking system. IEEE In INFOCOM.2000(2):775-784.

[4] Nissanka B. Priyantha, Anit Chakraborty, and Hari Balakrishnan. The Cricket Location-Support System.6th ACM Interantional Conference on Mobile Computing and Networking(ACM MOBICOM),Boston,MA,2000:32-43.

[5] Andy Harter, Andy Hopper, Pete Steggles et al.The Anatomy of a Context-Aware Application. Wireless Network 2001(1),1-16.

[6] J. Hightower, R. Want and G. Borriello,SpotON: An Indoor 3D Location Sensing Technology Based on RF Signal Strength. UW CSE Technical Report\#2000-02-02, WA(February 2000).

[7] LIONEL M.NI and YUNHAO LIU, YIU CHO LAU et al. LANDMARC: Indoor Location Sensing Using Active RFID. Wireless Networks 2004(10):701-710. 